\title{
Zur obligatorischen Teilnahme von muslimischen Schülerinnen am gemischten Schwimmunterricht
}

Eine Anmerkung zum Urteil des EGMR Osmanoğlu und Kocabaş gegen Schweiz

Sarah Progin-Theuerkauf *

Am 10. Januar 2017 entschied der Europäische Gerichtshof für Menschenrechte, dass die Verhängung einer Geldbusse in Höhe von 1'40o CHF, die die Erziehungsdirektion des Kantons Basel-Stadt gegen die Eltern zweier muslimischer Mädchen wegen wiederholter Verletzung der elterlichen Pflichten verhängt hatte, deren Recht auf Religionsfreiheit nach Art. 9 EMRK nicht verletzt hat. Der nachfolgende Beitrag fasst das Urteil, das nur in französischer Sprache erhältlich ist, zusammen und bewertet es.

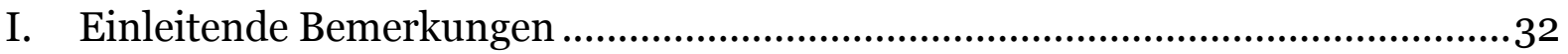

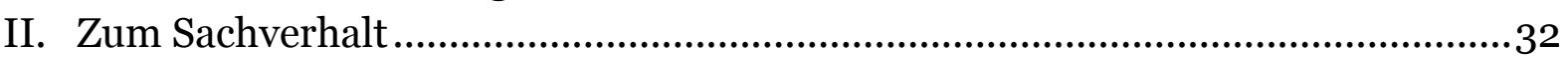

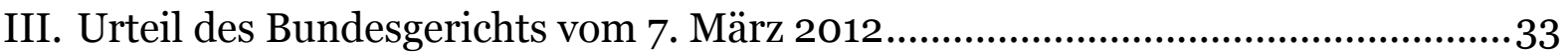

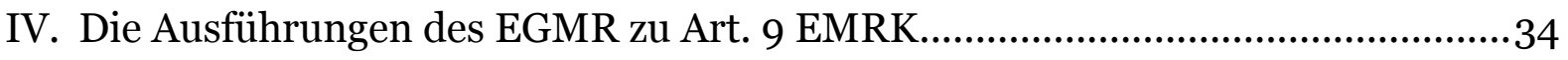

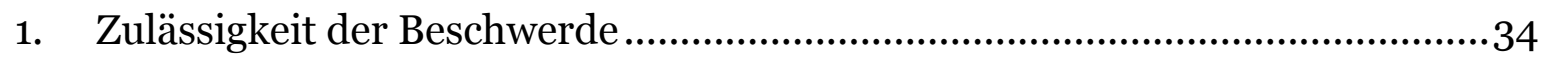

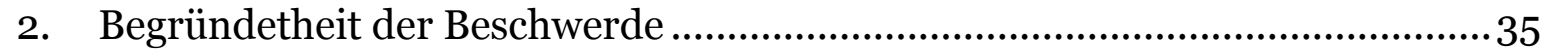

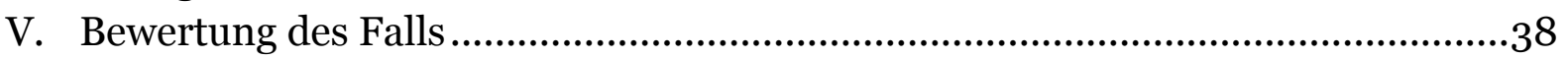

Zitiervorschlag: Sarah Progin-Theuerkauf, Zur obligatorischen Teilnahme von muslimischen Schülerinnen am gemischten Schwimmunterricht, in: sui-generis 2017, S. 31

URL: $\quad$ sui-generis.ch/31

DOI: $\quad$ https://doi.org/10.21257/sg.31

* Prof. Sarah Progin-Theuerkauf ist assoziierte Professorin für Europarecht und europäisches Migrationsrecht an der Universität Fribourg. Sie ist zudem Co-Direktorin des Zentrums für Migrationsrecht in Neuenburg.

Dieses Werk ist lizenziert unter einer Creative Commons Namensnennung - Weitergabe unter gleichen Bedingungen 4.0 International Lizenz. 


\section{Einleitende Bemerkungen}

1 Religiöse Bekleidungsvorschriften haben in der Vergangenheit schon oft Gesetzgeber und Gerichte in ganz Europa beschäftigt, Burkini-1 oder Burka-Verbot ${ }^{2}$ in Frankreich oder im Tessin3, sei es das Kopftuch bei Schülerinnen oder Lehrerinnen4. Im Januar 2017 wurde ein neuer Fall5 durch den Europäischen Gerichtshof für Menschenrechte (EGMR) entschieden; dieses Mal ging es allerdings nicht um das Tragen religiöser Kleidung, sondern um die Teilnahme muslimischer Kinder an bestimmten Schulfächern: Im Kanton Basel-Stadt wurden zwei muslimische Mädchen im Primarschulalter von ihren Eltern vom obligatorischen, gemischten Schwimmunterricht ferngehalten, weil dies mit ihrem muslimischen Glauben nicht vereinbar sei. Die Eltern wurden durch die Erziehungsdirektion des Kantons Basel-Stadt mit 1'400 CHF Bussgeld belegt, wogegen sie bis zum Bundesgericht erfolglos geklagt hatten. Der EGMR schloss sich dem Bundesge-

1 Vgl. nzz.ch vom 26. August 2016 (Staatsrat annulliert Burkini-Verbot).

2 Vgl. Urteil des EGMR 43835/11 vom 1. Juli 2014 (S.A.S. gegen Frankreich), RJD 2014-III.

3 Vgl. Art. 9a der Verfassung von Republik und Kanton Tessin sowie dessen einfachgesetzliche Umsetzung Vgl. auch Messaggio: Revisione totale della Legge sull'ordine pubblico del 29 maggio 1941 und srf.ch vom 1. Juli 2016 (Burka-Verbot im Tessin gilt ab heute) Die Änderungen sind seit dem 1. Juli 2016 in Kraft. Rekursen hiergegen wurde durch das Bundesgericht keine aufschiebende Wirkung gewährt, vgl. nzz.ch vom 15. Juli 2016 (Burka-Verbot im Tessin kann in Kraft treten).

4 Vgl. dazu die Zusammenfassung der Rechtsprechung auf humanrights.ch vom 19. Februar 2016 (Das Kopftuch an öffentlichen Schulen); siehe auch Astrid Epiney / Robert Mosters / Dominique Gross, Islamisches Kopftuch und religiöse Neutralität an der öffentlichen Schule, in: Pahud de Mortanges / Tanner (Hrsg.), Muslime und Schweizerische Rechtsordnung, 2002, S. 129.

5 Urteil des EGMR 29086/12 vom 10. Januar 2017 (Osmanoğlu und Kocabaş gegen Schweiz). richt $^{6}$ an und entschied einstimmig, dass eine Verletzung der Religionsfreiheit nach Art. 9 EMRK hier nicht feststellbar sei. Das Urteil wurde durch Experten und in den Medien mehrheitlich begrüsst.7 Es ist noch nicht rechtskräftig. ${ }^{8}$

\section{Zum Sachverhalt}

2 Die Beschwerdeführer (Herr Aziz Osmanoğlu, geboren 1976, und Frau Sehabat Kocabaş, geboren 1978), sind türkische Staatsangehörige, die in Basel leben. Der Beschwerdeführer war bereits im Alter von 10 Jahren in die Schweiz immigriert und zwischenzeitlich für ein islamwissenschaftliches Studium in die Türkei zurückgekehrt, wo er seine Ehefrau kennenlernte. Diese kam im Rahmen des Familiennachzugs 1999 in die Schweiz (vgl. Rz. 6 und $7^{9}$ ). Das Ehepaar hat drei

6 Urteil des Bundesgerichts 2C_666/2011 vom 7. März 2012. Vgl. auch bereits das Grundsatzurteil BGE 135 I 79 zu einem ähnlich gelagerten Fall aus dem Kanton Schaffhausen; dort allerdings ging es um Jungen im Primarschulalter. Anders noch BGE 119 Ia 178 ff. aus dem Jahr 1993, in dem ein Recht muslimischer Schülerinnen auf Befreiung vom Schwimmunterricht grundsätzlich anerkannt wurde. Dazu Hans Peter Moser, Bemerkungen zu BGE 119 Ia 178, ZBl 1994, 38; Sylvie Guichard, De la liberté de croyance à l'obligation de s'intégrer : les arrêts du Tribunal fédéral sur les dispenses de cours de natation pour des élèves musulmans, AJP 2014, 983.

7 Vgl. tagesanzeiger.ch vom 10. Januar 2017 (Musliminnen müssen in Schwimmunterricht); vgl. zeit.de vom 10. Januar 2017 (Das Recht, sich freizuschwimmen); vgl. nzz.ch vom 10. Januar 2017 (Keine muslimischen Sonderwünsche).

8 Nach Art. 44 Abs. 2 EMRK wird das Urteil einer Kammer endgültig, a) wenn die Parteien erklären, dass sie die Verweisung der Rechtssache an die Große Kammer nicht beantragen werden, b) drei Monate nach dem Datum des Urteils, wenn nicht die Verweisung der Rechtssache an die Große Kammer beantragt worden ist, oder c) wenn der Ausschuss der Großen Kammer den Antrag auf Verweisung nach Artikel 43 abgelehnt hat.

9 Der nachfolgende Text enthält eine leicht zusammengefasste Übersetzung des Sachverhalts 
Töchter, geboren 1999, 2001 und 2006. Die Beschwerde betrifft die beiden älteren Töchter, die zunächst dieselbe Primarschule in Basel besuchten. Die ältere der beiden Töchter wechselte später auf die Orientierungsschule (vgl. Rz. 8). Nach dem Schulgesetz Basel-Stadt (im Folgenden: SchulG) gehört der Schwimmunterricht zum obligatorischen Turnunterricht. SchülerInnen können vom Unterricht oder von einzelnen Unterrichtsfächern dispensiert werden; hierüber entscheidet die Schulleitung auf Antrag des Lehrpersonenteams oder auf Antrag der Erziehungsberechtigten. Den Beschwerdeführern wurde zudem unter Berufung auf eine Handreichung («Merkblatt zum Umgang mit religiösen Fragen an der Schule») mitgeteilt, dass eine Dispensation nur erteilt werden könne, wenn die betroffenen SchülerInnen Geschlechtsreife erlangt haben (vgl. Rz. 9).

3 Die Beschwerdeführer, strenggläubige Muslime, weigerten sich, ihre Töchter im Grundschulalter am gemischten Schwimmunterricht teilnehmen zu lassen. Sie beriefen sich darauf, ihr Glaube verbiete die Teilnahme an einem solchen gemischtgeschlechtlich erteilten Schwimmunterricht. Sie gaben zudem an, obschon der Koran die Bedeckung des weiblichen Körpers erst ab der Geschlechtsreife vorschreibe, geböte es ihr Glaube, ihre Töchter bereits vor der Pubertät auf die später für sie geltenden Vorschriften vorzubereiten. Als Erziehungsberechtigte der Töchter machten die Beschwerdeführer die Verletzung eigener Rechte geltend (vgl. Rz. 9).

unter «En fait», dort Teil I. A. des Urteils. Es wird am Ende jeden Abschnitts auf die entsprechenden Randziffern des Urteils verwiesen.
4 Im August 2008 wies das Erziehungsdepartement das Kantons Basel-Stadt die Beschwerdeführer darauf hin, dass sie nach § 91 Abs. 9 SchulG mit einer Ordnungsbusse bis $1^{\prime} \mathrm{OOO} \mathrm{CHF}$ belegt werden könnten, wenn ihre Töchter ihrer Pflicht zum Schulbesuch nicht nachkämen (vgl. Rz. 10).

$5 \quad$ Im März 2010 führte die Schulleiterin ein Gespräch mit den Beschwerdeführern, um nach einer Lösung zu suchen. Die Beschwerdeführer schickten ihre Töchter jedoch weiterhin nicht zum Schwimmunterricht. Im Mai 2010 wurden sie daher erneut aufgefordert, die Mädchen am Schwimmunterricht teilnehmen zu lassen. Dies geschah jedoch nicht (vgl. Rz. 11).

6 Im Mai und Juni 2010 beantragte die Schulleitung beim Departementsvorsteher des Erziehungsdepartements, ein Ordnungsbussenverfahren gegen die Beschwerdeführer einzuleiten. Im Juni 2010 wurde den Beschwerdeführern in diesem Rahmen Gelegenheit gegeben, sich zu äussern (vgl. Rz. 12).

7 Im Juli 2010 büsste das Erziehungsdepartement die beiden Eltern wegen wiederholter Verletzung der elterlichen Pflichten mit 350 CHF pro Tochter pro Elternteil, insgesamt also 1'400 CHF. Den hiergegen erhobenen Rekurs der Eltern wies das Appellationsgericht des Kantons Basel-Stadt im Mai 2011 ab (vgl. Rz. 13 und 14).

\section{Urteil des Bundesgerichts vom} 7. März 2012

8 Auch das Bundesgericht wies die Beschwerde in öffentlich-rechtlichen Ange- 
legenheiten der Beschwerdeführer im März $2012 \mathrm{ab}^{10}$. Es kam in seinem Urteil zu dem Ergebnis, eine Verletzung der Glaubens- und Gewissensfreiheit der Beschwerdeführer ${ }^{11}$ liege nicht vor. Zwar bestehe ein Eingriff in die Glaubens- und Gewissensfreiheit, dieser beruhe aber auf einer hinreichenden gesetzlichen Grundlage (nämlich dem Schulgesetz in Verbindung mit dem kantonalen Lehrplan ${ }^{12}$; die entsprechende Handreichung sei nur eine Anleitung zur Handhabung des Ermessens bei der Erteilung von Dispensationen) (vgl. Rz. 16 bis 18).

9 Was das erforderliche öffentliche Interesse und die Verhältnismässigkeit angeht, so bestätigte das Bundesgericht die Argumentation der Vorinstanz: Es bestehe ein überwiegendes öffentliches Interesse an einem obligatorischen Schwimmunterricht und der Integration aller Schüler, unabhängig von ihrer Herkunft, Kultur und Religion. Der Eingriff sei auch nicht unverhältnismässig. Der gemischt-geschlechtliche Schwimmunterricht finde nur für Kinder vor der Geschlechtsreife statt und werde durch flankierende Massnahmen (getrennte Umkleide- und Duschräume, Verwendung von körperbedeckenden Schwimmanzügen) gemildert. Es gehe schliesslich nicht allein um den Inhalt des Lernstoffs, sondern auch um die äusseren Bedingungen des Unterrichts. Die soziale Einbindungsfunktion der Schule erfordere, dass sie für alle obligatorisch sei und Dispensationen nur mit Zurückhaltung erteilt würden. Die neue Praxis des Bundesgerichts betone den grundsätzlichen

\footnotetext{
10 Vgl. Fn. 6.

11 Vgl. Art. 15 BV, der im Urteil des EGMR nicht zitiert wird.

12 Vgl. BGE 135 I 79 E. 6.
}

Vorrang der schulischen Pflichten vor der Beachtung religiöser Gebote einzelner Bevölkerungsteile. Aus diesem Grund gehe auch der Vergleich mit Dispensationen aus gesundheitlichen Gründen fehl (vgl. Rz. 19 bis 21).13

\section{Die Ausführungen des EGMR zu Art. 9 EMRK}

10 Der EGMR behandelt zunächst die Frage der Zulässigkeit der Beschwerde (vgl. Rz. 35 und 36) und prüft sodann eine mögliche Verletzung der Religionsfreiheit (vgl. Rz. 37 bis 106).

\section{Zulässigkeit der Beschwerde}

11 Der EGMR weist zunächst darauf hin, dass Art. 2 S. 2 des 1. Zusatzprotokolls (ZP) zur EMRK ${ }^{14}$ im Vergleich zu Art. 9 EMRK lex specialis sei, da dieser explizit das «Recht der Eltern, die Erziehung und den Unterricht entsprechend ihren eigenen religiösen und weltanschaulichen Überzeugungen sicherzustellen» beinhalte. Die Schweiz habe das 1. ZP zur EMRK aber nicht ratifiziert; daher könnten die Beschwerdeführer sich dennoch auf Art. 9 EMRK berufen. Die Situation der Beschwerdeführer falle auch grundsätzlich in den Anwendungsbereich von Art. 9 EMRK. Die Beschwerde sei nicht offensichtlich unbegründet (Art. 35 Abs. 3a

13 Das Bundesgericht geht in seinem Urteil 2C_666/2011 vom 7. März 2012 noch ausführlicher auf die etwa drei Jahre zuvor erfolgte Rechtsprechungsänderung (vgl. Fn. 6) ein und erklärt, dass es für eine erneute Rechtsprechungsänderung gewichtiger Gründe bedürfe, die hier aber nicht ersichtlich seien (vgl. E. 2.6.3. des Urteils). Dieser Punkt wird im Urteil des EGMR allerdings nicht erwähnt. Vgl. auch die Bewertung des Falls (V.).

14 Im Internet abrufbar unter coe.int (Zusatzprotokoll zur Konvention zum Schutze der Menschenrechte und Grundfreiheiten). 
EMRK) und somit zulässig (vgl. Rz. 35 und 36).

\section{Begründetheit der Beschwerde}

12 In materieller Hinsicht stellt der EGMR fest, dass es sich im Ausgangsfall um eine Situation handele, in dem die Religionsausübungsfreiheit der Beschwerdeführer betroffen sei. Diese hätten die elterliche Sorge inne und dürften damit über die religiöse Erziehung ihrer Kinder bestimmen. Sie könnten sich daher auf diesen Teilaspekt von Art. 9 EMRK berufen. Der Gerichtshof ist ferner der Ansicht, die Beschwerdeführer hätten einen Eingriff in ihr Recht auf Religionsfreiheit erlitten (Rz. 37 bis 42).

In einem nächsten Schritt prüft der EGMR, auf welcher Rechtsgrundlage der Eingriff erfolgt ist. Hier kommt der Gerichtshof zum Ergebnis, diese sei im im Internet abrufbaren - Lehrplan sowie im entsprechenden Schulgesetz zu sehen. Zudem seien die Beschwerdeführer durch das Erziehungsdepartement auf eine mögliche Busse in Höhe von maximal 1'ooo CHF hingewiesen worden. Der Eingriff in ihr Grundrecht auf Religionsfreiheit sei damit auch vorhersehbar gewesen (vgl. Rz. 43 bis 55).

Was den mit der Massnahme verfolgten legitimen Zweck angeht, so folgt der EGMR hier der Einschätzung der Schweizer Regierung: Die strittige Massnahme habe die Integration von Kindern aus verschiedenen Kulturen und Religionen sowie den ordnungsgemässen Ablauf des Unterrichts, die Einhaltung der Schulpflicht und die Gleichstellung der Geschlechter zum Zweck (vgl. Rz. 56 bis 65).
15 Die Massnahme sei schliesslich auch notwendig in einer demokratischen Gesellschaft (vgl. Rz. 66 bis 106). In diesem Zusammenhang ruft der EGMR zunächst die Bedeutung von Art. 9 EMRK in Erinnerung und äussert einige allgemeine Grundprinzipien. Die Gedanken-, Gewissens- und Religionsfreiheit gehöre zum Fundament der «demokratischen Gesellschaft» im Sinne der Konvention. Diese Freiheit sei Teil der wesentlichen Elemente der Identität von Gläubigen und ihrer Lebensauffassung, aber auch für Atheisten, Agnostiker, Skeptiker und Gleichgültige ein wichtiges Gut. ${ }^{15}$ Die Religionsfreiheit schütze nicht nur das forum internum, sondern umfasse auch die Freiheit, seine Religion individuell, privat, kollektiv, öffentlich oder im Kreis anderer Gläubiger kundzutun. Art. 9 EMRK nenne verschiedene Formen des Glaubensbekenntnisses, etwa den Gottesdienst, den Unterricht, oder das Praktizieren von Bräuchen und Riten. Allerdings werde nicht jede religiös inspirierte Handlung geschützt und das Recht, sich in der Öffentlichkeit gemäss seines Glaubens zu verhalten, sei nicht absolut (vgl. Rz. 82 und 83).

16 Pluralismus, Toleranz und Weltoffenheit seien ebenso Charaktermerkmale einer «demokratischen Gesellschaft». Auch wenn man sich als Individuum manchmal den Interessen einer Gruppe unterordnen müsse, garantiere eine Demokratie nicht die ständige Vorherrschaft der Mehrheitsmeinung, sondern verlange darüber hinaus eine gerechte Behandlung von Minderheiten und die Vermeidung des Missbrauchs einer beherrschenden Stellung (vgl. Rz. 84).

15 Urteil des EGMR 62649/10 vom 26. April 2016 (İzzettin Doğan u.a. gegen Türkei), Rn. 103. 
17 Ein Staat müsse nicht nur Eingriffe in die durch die Konvention geschützten Rechte vermeiden, sondern habe u.U. auch positive Verpflichtungen, z.B. das Schaffen eines effektiven und zugänglichen Verfahrens, um die durch die Konvention geschützten Rechte durchzusetzen. Der Mechanismus der Konvention habe nur subsidiären Charakter. Nationale Gerichte seien in der Regel viel besser in der Lage, örtliche Bedürfnisse und Zusammenhänge zu bewerten (vgl. Rz. 85).

Der EGMR habe bereits Gelegenheit gehabt, festzuhalten, dass es in Europa keine einheitliche Auffassung über die Bedeutung von Religion gebe und dass Bedeutung oder Auswirkungen von Handlungen, die öffentlicher Ausdruck einer bestimmten religiösen Überzeugung sind, je nach Epoche und Kontext variieren könnten. ${ }^{16}$ Gesetzliche Regelungen könnten daher auch von einem Land zum anderen unterschiedlich sein. Der entsprechende Beurteilungsspielraum werde aber durch den EGMR beschränkt, der sowohl ein Gesetz als auch die auf ihm beruhenden Entscheidungen in der Praxis überprüfen könne. Insbesondere könne geprüft werden, ob eine nationale Massnahme gerechtfertigt sei und den Grundsatz der Verhältnismässigkeit wahre. Der Gerichthof müsse den ihm vorgelegten Fall stets im Lichte des Gesamtzusammenhangs beurteilen (vgl. Rz. 88 und 89).

19 Die Schweiz habe das ZP 1 zur Konvention nicht ratifiziert, daher beriefen sich die Beschwerdeführer hier auf Art. 9 EMRK. Der Gerichtshof müsse den Fall daher nach diesem Artikel beurteilen. Al-

16 Urteil des EGMR 44774/98 vom 10. November 2005 (Leyla Şahin gegen Türkei), RJD 2005-XI. lerdings halte es der EGMR für nützlich, auch die im Rahmen von Art. 2 ZP 1 entwickelten Grundsätze in Erinnerung zu rufen, da die Konvention als Ganzes gelesen werden müsse und da der zweite Satz von Art. 2 ZP 1 gegenüber Art. 9 EMRK lex specialis sei. Dieser knüpfe an das in Art. 2 Satz 1 ZP 1 verankerte Recht auf Bildung an. In erster Linie sei es demnach Recht der Eltern, die Erziehung und den Unterricht ihrer Kinder zu gewährleisten. In Ausübung dieses Rechts könnten die Eltern vom Staat die Wahrung ihrer eigenen religiösen und weltanschaulichen Überzeugungen verlangen. Der zweite Satz impliziere, dass der Staat einen gewissen erzieherischen Pluralismus garantiere, der für den Erhalt einer demokratischen Gesellschaft unabdingbar sei. Der Staat müsse daher auf eine objektive, kritische und pluralistische Erziehung achten und dürfe keine Indoktrinierung betreiben. Der Begriff «achten» in Art. 2 ZP 1 verlange mehr als als ein blosses «Anerkennen» oder «Berücksichtigen»; er erfordere zusätzlich zu negativen Bemühungen auch eine bestimmte positive Verpflichtung. In dieser Hinsicht habe der Staat jedoch einen breiten Ermessensspielraum. Eltern könnten daher vom Staat nicht die Durchführung eines bestimmten Unterrichts verlangen (vgl. Rz. 90 bis 92).

20 Schliesslich wiederholt der Gerichtshof die bekannte Formel, Ziel der EMRK sei, die in ihr verankerten Rechte konkret und effektiv, und nicht bloss theoretisch und illusorisch ${ }^{17} \mathrm{zu}$ schützen (vgl. Rz. 92).

17 Vgl. Urteil des EGMR 15472/02 vom 29. Juni 2007 (Folgerø u.a. gegen Norwegen), RJD 2007III, Rn. 100; Urteil des EGMR 30985/96 vom 26. Oktober 2000 (Hassan und Tchaouch gegen Bulgarien), RJD 2000-XI, Rn. 62; Urteil des EGMR 76836/01 und 32782/03 vom 1. Oktober 
Er widmet sich sodann der Untersuchung, ob im konkreten Fall die Weigerung der zuständigen Behörden, die Töchter der Beschwerdeführer vom gemischtgeschlechtlichen Schwimmunterricht zu dispensieren, in einer demokratischen Gesellschaft notwendig und verhältnismässig war (vgl. Rz. 95 ff.).

Hierbei sei zu berücksichtigen, dass die EMRK-Mitgliedstaaten über einen Ermessensspielraum verfügten, was Fragen der Beziehungen zwischen dem Staat und den Religionen sowie der Bedeutung, der der Religion in der Gesellschaft zukommt, angehe, dies umso mehr, als es sich hier um Fragen der Erziehung und des öffentlichen Unterrichts handele. Staaten seien darüber hinaus frei, Lehrpläne entsprechend ihren Bedürfnissen und Traditionen zu erstellen. Eltern hätten zwar das Recht, über die Erziehung ihrer Kinder zu bestimmen, jedoch könnten sie nicht von einem Staat verlangen, dass dieser eine bestimmte Art des Unterrichts anbiete oder diesen auf eine bestimmte Art und Weise aufbaue. Diese Grundsätze seien umso wichtiger für die vorliegende Beschwerde, als die Schweiz das ZP 1 zur EMRK nicht ratifiziert habe und föderal organisiert sei, weshalb Kantonen und Gemeinden umfangreiche Kompetenzen im Bereich des Aufbaus und der Gestaltung von Lehrplänen zukämen (vgl. Rz. 95).

Was die Abwägung der betroffenen Interessen angeht, so hält der EGMR die Argumente der Schweizer Regierung für überzeugend: Der Schule komme eine besondere Rolle im Rahmen des Prozesses sozialer Integration zu; dies gelte umso mehr in Bezug auf ausländische Kinder. Angesichts der Bedeutung der obligatorischen Schulbildung sei die Gewährung von Dispensationen nur in besonderen Ausnahmefällen unter klaren Bedingungen und unter Beachtung der Gleichbehandlung aller religiöser Gruppen gerechtfertigt. Die Tatsache, dass eine Ausnahme aus medizinischen Gründen möglich sei, zeige, dass hier keine exzessive Härte angewendet werde. Auch wenn nur sehr wenige Eltern eine Ausnahme beantragten, habe hier das Interesse der Kinder an einer vollständigen Schulbildung, die ihnen eine erfolgreiche soziale Integration entsprechend den vor Ort geltenden Sitten und Gebräuchen ermögliche, Vorrang vor dem Interesse der Eltern an einer Dispensation ihrer Töchter vom gemischtgeschlechtlichen Schwimmunterricht (vgl. Rz. 96 und 97).

23 Auch wenn der Schwimmunterricht nicht an allen Schweizer Schulen Teil des obligatorischen Schulstoffs sei (nicht einmal innerhalb des Kantons Basel-Stadt), so sei die Sporterziehung besonders wichtig für die Entwicklung und die Gesundheit der Kinder. Es gehe hier auch nicht allein um die Tatsache, das Schwimmen als solches zu erlernen, sondern auch und gerade um das Lernen und Ausüben einer körperlichen Aktivität gemeinsam mit anderen SchülerInnen, ohne Unterschied in Hinblick auf Herkunft der Kinder oder religiöse und weltanschauliche Überzeugungen der Eltern (vgl. Rz. 98).

2009 (Kimlya u.a. gegen Russland), RJD 2009IV, Rn. 86; Urteil des EGMR 6694/74 vom 13. Mai 1980 (Artico gegen Italien), Serie A37, Rn. 33. 
24 Der Gerichtshof weist weiter darauf hin, dass er auf die Besonderheiten eines föderalen Staates Rücksicht nehme. ${ }^{18}$ Dass eine schweizweite, einheitliche Regelung hier nicht existiere, könne nicht als Argument gelten. Würde man schliesslich Kindern eine Ausnahme gewähren, deren Eltern ihnen privaten Schwimmunterricht finanzierten, so würde dies Kinder diskriminieren, deren Eltern sich dies nicht leisten könnten (vgl. Rz. 99 und 100).

Weiterhin betont der EGMR, die im Fall zuständigen Behörden hätten den Beschwerdeführern bedeutende Zugeständnisse angeboten, namentlich das Tragen eines Burkinis durch die beiden Mädchen. Schliesslich hätten für die Mädchen getrennte Duschen und Umkleideräume zur Verfügung gestanden. Zum Burkini hatten die Eltern jedoch argumentiert, dies stigmatisiere ihre Töchter, was allerdings nicht weiter belegt wurde. Alles in allem war der Gerichtshof der Ansicht, die angebotenen Massnahmen seien geeignet, die Auswirkungen der Pflicht zur Teilnahme am Schwimmunterricht auf die religiösen Überzeugungen der Eltern deutlich abzumildern (vgl. Rz. 101).

Schliesslich seien auch keine weiteren Beschränkungen in der Ausübung oder Bekundung der religiösen Überzeugungen der Mädchen erkennbar. ${ }^{19}$ Auch was die Höhe der verhängten Bussen angeht, so sei hier nicht von einer Unverhältnismässigkeit auszugehen. Schliesslich liege

18 Vgl. etwa Urteil des EGMR 16354/o6 vom 13. Juli 2012 (Mouvement Raelien Suisse gegen Schweiz), RJD 2012 (Auszüge), Rn. 64. Weitere Nachweise im Urteil unter Rz. 99.

19 Vgl. Urteil des EGMR 30814/06 vom 18. März 2011 (Lautsi u.a. gegen Italien), RJD 2011-III. auch keine Verletzung von Art. 9 EMRK aufgrund von fehlenden Verfahren zur Überprüfung der durch die Beschwerdeführer kritisierten Entscheide vor (vgl. Rz. 102 bis 104).

27 Dementsprechend sei insgesamt keine Verletzung von Art. 9 EMRK feststellbar (vgl. Rz. 105 und 106).

\section{Bewertung des Falls}

28 Insgesamt erscheint das Urteil im Fall Osmanoğlu und Kocabaş überzeugend: Weder richtet es sich gegen den Islam als solchen, noch bekräftigt es einen Vorrang der in Europa vorherrschenden christlich-abendländischen Traditionen. Im Gegenteil: Es wird klargestellt, dass sich alle religiösen Gruppen denselben Grundsätzen zu unterwerfen haben. Die allgemeine Schulpflicht soll es allen Kindern in einer Gesellschaft ermöglichen, verschiedene Lebensweisen und Religionen kennenzulernen und innerhalb der Schul- bzw. Klassengemeinschaft gemeinsam mit Altersgenossen diejenigen Kenntnisse zu erwerben, die der Staat für wichtig hält. Hierbei verfügt ein Staat über einen erheblichen Ermessensspielraum. Dieser ist auch vor dem Hintergrund zu sehen, dass die Mitgliedstaaten der EMRK nicht alle in der gleichen Art und Weise das Verhältnis von Staat und Religion geregelt haben. Die Bedeutung, die der Religion in der betroffenen Gesellschaft zukommt, kann also durchaus unterschiedlich gesehen und dementsprechend Eingriffe in die Religionsfreiheit unterschiedlich bewertet werden.

29 Ein Staat hat also in doppelter Hinsicht Spielraum: Zum einen hinsichtlich der Bedeutung, die er Religionen (oder einer 
bestimmten Religion) zuerkennt, zum anderen hinsichtlich des Wissens, das Kinder im Rahmen der allgemeinen Schulpflicht erwerben sollen. Insofern beschränkt sich der Gerichtshof hier richtigerweise ausschliesslich darauf, zu überprüfen, ob die Abwägungsentscheidung zwischen privatem Interesse und öffentlichem Interesse fehlerhaft war. Das in diesem Fall einschlägige private Interesse, das Erziehungsrecht der Eltern in religiösen Fragen, tritt im vorliegenden Fall gegenüber dem öffentlichen Interesse an der Integration aller Kinder zurück.

Die Integrationspflicht trifft dabei selbstredend nicht nur Muslime, sondern auch Angehörige anderer Glaubensgemeinschaften. Alle müssen in bestimmten Situationen, wie beim Schulbesuch, eine gewisse Anpassung ihrer Gewohnheiten akzeptieren. Der Schwimmunterricht ist nur ein Beispiel von vielen, bei dem verschiedene Auffassungen aufeinander treffen. Hier besteht inzwischen seitens der Schulen relativ viel Toleranz, was religiöse Bekleidungsvorschriften angeht (Tragen eines Burkinis). Mit Dispensationen wird aber richtigerweise zurückhaltend umgegangen, um die betroffenen Kinder nicht $\mathrm{zu}$ isolieren. ${ }^{20}$

Insofern ist die Rechtsprechungsänderung, die das Bundesgericht im Oktober 2008 vollzogen hat, begrüssenswert. ${ }^{21} \mathrm{Im}$ Gegensatz zur seiner früheren Rechtsprechung22 betonte das Bundesgericht

20 Vgl. Urteil des EGMR 35504/O3 vom 11. September 2006 (Konrad u.a. gegen Deutschland), RJD 2006-XIII (Heimunterricht); Urteil des EGMR 319/08 vom 13. September 2011 (Dojan gegen Deutschland) (Sexualkundeunterricht).

21 BGE 135 I 79 (vgl. Fn. 6).

22 BGE 119 Ia 178 (vgl. Fn. 6). in diesem Urteil, «das Obligatorium des Schulbesuches» - einschliesslich der vom kantonalen Recht statuierten Pflicht zur Teilnahme am Schwimmen im Rahmen des Sportunterrichts - diene «der Wahrung der Chancengleichheit aller Kinder und darüber hinaus auch derjenigen zwischen den Geschlechtern bzw. der Gleichstellung von Mann und Frau in der (Aus-)Bildung»; sie fördere zudem «die Integration von Angehörigen anderer Länder, Kulturen und Religionen und ist somit unbestrittenermassen von gewichtigem öffentlichen Interesse». ${ }^{23}$ Dem gemeinsam geführten Sportunterricht komme im in der Schweiz bestehenden gesellschaftlichen Umfeld zudem eine im Interesse des Kindes liegende - wichtige sozialisierende Funktion zu. Insbesondere gelte es zu vermeiden, dass Kinder islamischen Glaubens bereits auf der Schulstufe in eine Aussenseiterrolle gedrängt werden. Integrationsanliegen hätten in der Öffentlichkeit in den vergangenen Jahren noch mehr Gewicht erhalten. Auch die religiöse Zusammensetzung der Schweizer Wohnbevölkerung habe sich verändert. Die inzwischen bestehende «multikulturelle Schulrealität» verlange vermehrt Anstrengungen zur Angewöhnung und Einbindung der Kinder und Jugendlichen aus anderen Kulturen in die hier geltenden gesellschaftlichen Rahmenbedingungen. ${ }^{24}$ Diesen Ausführungen ist zuzustimmen. ${ }^{25}$

32 Da im Ausgangsfall nur der Randbereich der Religionsausübungsfreiheit betroffen ist, überwiegt also das öffentliche Inte-

BGE 135 I 79 E. 7.1.

24 BGE 135 I 79 E. 7.2.

25 Das Bundesgericht sagt denn auch in seinem Urteil 2C_666/2011 vom 7. März 2012: «Die Erwägungen zur Integration muslimischer Bevölkerungskreise gelten nach wie vor.» 
resse an der Gleichbehandlung und Integration aller Kinder. Es ist aber richtig, hier je nach Tragweite der den Kindern auferlegten Massnahmen zu differenzieren: Den eigenen Körper dem entgegengesetzten Geschlecht nicht zeigen zu wollen, ist in der Regel (mindestens ab der Pubertät) von der Religionsfreiheit gedeckt. Andere nicht sehen zu wollen hingegen nicht... Es soll schliesslich betont werden, dass der EGMR die Glaubensvorstellungen der Beschwerdeführer an keiner Stelle bewertet oder auf ihre theologische Richtigkeit hin untersucht. ${ }^{26}$ Insofern ist das Urteil des EGMR sehr klar und abstraktionsfähig.

Letztlich muss auch bedacht werden, dass es auch den strengsten Eltern nicht möglich sein dürfte, ihre Kinder völlig vor der Konfrontation mit dem in der Schweiz und den meisten europäischen Staaten herrschenden, eher ungezwungen-freizügigen Umgang von Jungen und Mädchen (und später Männern und Frauen) «zu bewahren». Dazu müsste man die Kinder (in der Regel Mädchen) wohl einsperren, da diese schon bei einem blossen Spaziergang an einem See, beim Zappen im TV oder beim Vorbeigehen an einem Zeitschriftenkiosk dem Anblick teilweise entblösster Körper ausgesetzt sind. ${ }^{27}$ Dafür, dass Kinder durch ihre Eltern nicht völlig isoliert werden, kann der Staat wenigstens im Rahmen der öffentlichen Schulen sorgen, an denen alle Kinder den gleichen Lernbedingungen unterworfen sind und in denen es ihnen möglich gemacht wird, auch andere Auffassungen kennenzulernen, als

26 Dies hat auch das Bundesgericht so gehandhabt, vgl. BGE 135 I 79 E. 4.4., mit Verweis auf BGE 119 Ia 178 E. $4 \mathrm{c}$.

27 Dieses Argument wurde auch durch die Schweizer Regierung vorgetragen, vgl. Rz. 77 des Urteils. die der eigenen Eltern. Insbesondere soll den Kindern vermittelt werden, dass Gleichbehandlung und Toleranz gegenüber Andersartigem Grundpfeiler unserer Gesellschaft sind. Das Urteil leistet schliesslich nicht nur einen wichtigen Beitrag zur Integration ausländischer Kinder, sondern auch zur Gleichstellung von Mann und Frau. ${ }^{28}$ Dieser Aspekt ist, obschon er durch den EGMR nicht thematisiert wurde, nicht unbedeutend, da sich viele religiöse Vorschriften in erster Linie an Frauen richten. ${ }^{29}$ Es ist aber gerade Pflicht der Schule, Mädchen und Jungen dieselbe Ausbildung zu ermöglichen. Daher ist gerade beim Umgang mit Dispensationen, die in der Regel Mädchen betreffen, Vorsicht geboten.

34 Nicht zuletzt fällt auf, dass der Gerichtshof im Rahmen der Verhältnismässigkeitsprüfung in Rz. 90 ff. vertieft auf Art. 2 ZP 1 eingeht, obgleich die Schweiz das ZP 1 nicht ratifiziert hat. Er stellt dabei zwar klar, dies sei nur der Vollständigkeit halber (vgl. Rz. 90: «dans le souci d'être complet»). Dennoch betont er, die Konvention müsse «als Ganzes» gesehen werden. Insofern wendet der EGMR in der Prüfung von Art. 9 dieselben Grundsätze an, wie sie im Rahmen von Art. 2 ZP 1 entwickelt wurden.

28 Vgl. dazu grundlegend Walter Kälin, Grundrechte im Kulturkonflikt, Freiheit und Gleichheit in der Einwanderungsgesellschaft, 2000; Gianfranco Helbling, Das völkerrechtliche Verbot der Geschlechterdiskriminierung in einem plurikulturellen Kontext, Das Beispiel des Schutzes der Menschenrechte muslimischer Frauen in westlichen Ländern, 2001; Walter Frenz, Glaubensfreiheit und Schulpflicht, Juristische Ausbildung, 2013, 999.

29 Es ist jedoch nicht ausgeschlossen, dass davon auch Jungen betroffen sein könnten, wie BGE 135 I 79 gezeigt hat. 
35 Immerhin betont der EGMR aber, dass der Schweiz aufgrund der NichtRatifizierung des ZP 1 ein noch grösserer Ermessensspielraum zustehe, was das Gestalten der Inhalte der obligatorischen Schule angehe (Rz. 95). Da sich der Gehalt der beiden Grundrechte der Religionsfreiheit sowie des elterlichen Erziehungsrechts gemäss den eigenen religiösen Überzeugungen in diesem Aspekt überschneidet und Art. 2 ZP 1 lex specialis zu Art. 9 EMRK (vgl. Rz. 90) ist, ist gegen diese Auslegung nichts einzuwenden. Sofern ein Staat also das ZP 1 nicht ratifiziert hat, ist dieser Teilgehalt des Rechts auf Bildung von der Religionsfreiheit erfasst. 30

30 Auch im Bundesgerichtsurteil (Fn. 6) wurde mit der Glaubens- und Gewissensfreiheit (Art. 15 BV) argumentiert, da die BV ein Art 2 ZP 1 entsprechendes Grundrecht nicht enthält. 